\title{
On the Behavior of the Sierpinski Multiband Fractal Antenna
}

\author{
Carles Puente-Baliarda, Member, IEEE, Jordi Romeu, Member, IEEE, \\ Rafael Pous, Member, IEEE, Angel Cardama, Member, IEEE
}

\begin{abstract}
The multiband behavior of the fractal Sierpinski antenna is described in this paper. Due to its mainly triangular shape, the antenna is compared to the well-known single-band bow-tie antenna. Both experimental and numerical results show that the self-similarity properties of the fractal shape are translated into its electromagnetic behavior. A deeper physical insight on such a behavior is achieved by means of the computed current densities over the antenna surface, which also display some similarity properties through the bands.
\end{abstract}

Index Terms-Antennas, fractals, multifrequency antennas.

\section{INTRODUCTION}

$\mathbf{T}$ HE interaction of electromagnetic waves with fractal bodies has been recently studied [1]-[7]. Most fractal objects have self-similar shapes, which means that some of their parts have the same shape as the whole object but at a different scale [8]-[11]. The construction of many ideal fractal shapes is usually carried out by applying an infinite number of times an iterative algorithm such as the multiple reduction copy machine (MRCM) algorithm [8]. In such iterative procedure, an initial structure called generator is replicated many times at different scales, positions and directions, to grow the final fractal structure. D. L. Jaggard et al. [3] showed that the same kind of geometrical similarity relations at several growth stages were found in the electromagnetic behavior of the fractal body. The diffraction of fractally serrated apertures [5], [6] the reflection and transmission coefficients of fractal multilayers [7] and the sidelobe properties of some fractal arrays [2], [12], [13] are other examples of studies currently available in the literature that relate fractals and electromagnetics.

A first attempt to explore the multifrequency properties of fractals as radiating structures was done in [13]. The aim in that paper was to essay a new set of shapes for the design of multifrequency antenna arrays. Wide-band and frequencyindependent antennas were developed and thoroughly analyzed in the early sixties [14]-[19] and some convoluted shapes were investigated to try to elude the principle of the antenna radiation parameters dependence on its physical size relative

Manuscript received September 18, 1996; revised September 8, 1997. This work was supported in part by Grant TIC-96-0724-C06-04 from the Spanish Government and by the company FRACTUS S.A.

C. Puente-Baliarda is with the Department of Signal Theory and Communications (TSC), Polytechnic University of Catalonia, Barcelona, Spain.

J. Romeu and R. Pous are with the Polytechnic University of Catalonia, Barcelona, Spain.

A. Cardama is with the Telecommunication Engineering School, Polytechnic University of Catalonia, Barcelona, Spain.

Publisher Item Identifier S 0018-926X(98)02678-7. to wavelength. Spirals and log-periodic structures are some examples of successful structures used to design frequencyindependent antennas. Fractals might join some of those early designs due to their self-scaling properties [8]. Concerning that particular issue, Puente et al. described in [20] the behavior of, at the extent known by the authors, the first fractal multiband antenna-the Sierpinski monopole. Such a monopole displayed a similar behavior at several bands from both the input return loss and radiation patterns points of view. Some steps further in the field of multiband fractal antennas were done later in [21]-[23]. Furthermore, other interesting contributions regarding small [24] and frequency-independent [25] fractal antennas have been introduced by Cohen et al., respectively.

In this paper, the behavior of both the Sierpinski monopole and dipole is described by means of experimental and computational results and the comparison with the triangular (bowtie) antenna is done. The radiation pattern of the measured fractal dipole clearly shows a better resemblance at different frequencies than those of the monopole confirming, thus, that early disagreements among patterns were due to the finite size of the conductor ground plane. Also, the electric current density distribution over the fractal structure has been computed, giving some insight on the multifrequency behavior of the antenna.

\section{THE SIERPINSKI MONOPOLE}

\section{A. Antenna Description}

The Sierpinski gasket is named after the Polish mathematician Sierpinski who described some of the main properties of this fractal shape in 1916 [8], [26]. The original gasket is constructed by subtracting a central inverted triangle from a main triangle shape (Fig. 1). After the subtraction, three equal triangles remain on the structure, each one being half of the size of the original one. One can iterate the same subtraction procedure on the remaining triangles and if the iteration is carried out an infinite number of times, the ideal fractal Sierpinski gasket is obtained. In such an ideal structure, each one of its three main parts is exactly equal to the whole object, but scaled by a factor of two and so are each of the three gaskets that compose any of those parts. Due to this particular similarity properties, shared with many other fractal shapes, it is said that the Sierpinski gasket is a self-similar structure [23]. 


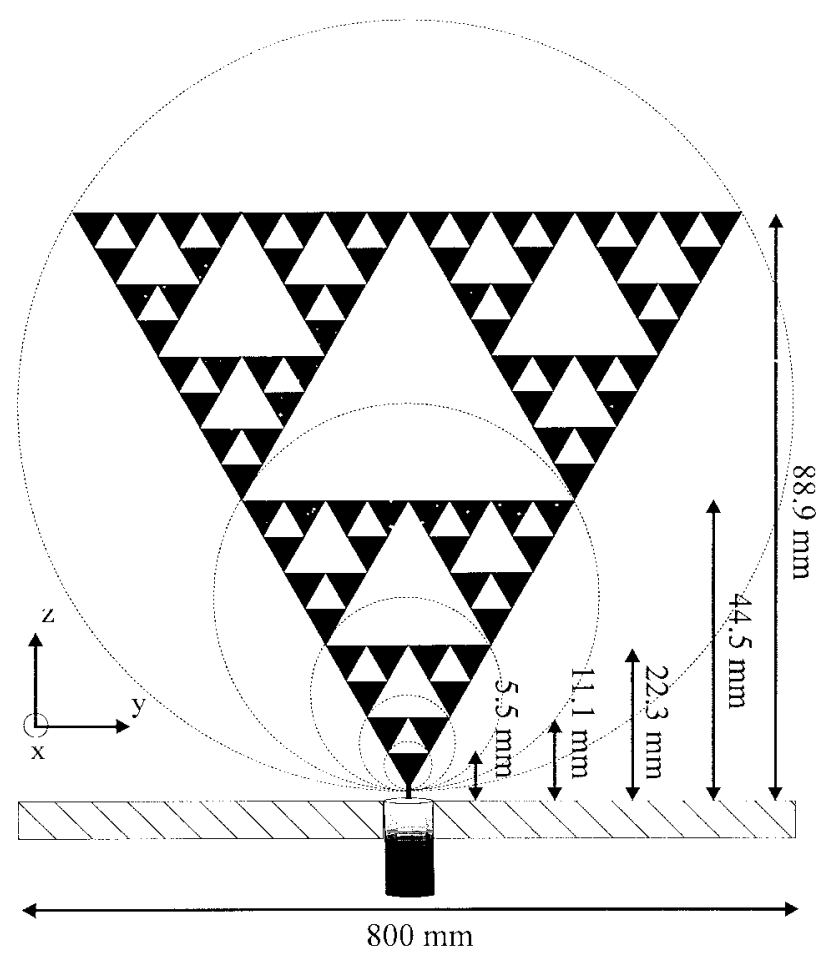

Fig. 1. The Sierpinski monopole. Each subgasket (circled) is a scaled version of the whole structure.

The Sierpinski gasket (also Sierpinski triangle) was chosen as the first candidate for a fractal antenna due to its resemblance to the triangular or bow-tie antenna. As shown in Fig. 1, the gasket was printed on a 1.588-mm-thick Cuclad 250 substrate $\left(\varepsilon_{r}=2.5\right)$ and mounted over a $800 \times 800$ $\mathrm{mm}$ ground plane. The structure was fed through a $1.5-\mathrm{mm}$ diameter, $50-\Omega$ coaxial probe with an SMA connector on the bottom side of the plane. The antenna is a scaled version of the antenna described in [20] with a thicker substrate to provide the printed fractal a stiffer support.

The gasket has been constructed through five iterations in this particular case, so five-scaled versions of the Sierpinski gasket are found on the antenna (circled regions in Fig. 1), the smallest one being a single triangle. If one neglects the contribution of the center holes to the antenna performance and admits that the current flowing from the feeder should concentrate over a region that is comparable in size to the wavelength, a behavior similar to five-scaled bow-tie antennas (each one operating at its resonant frequency) could be expected. The scale factor among the five gaskets is $\delta=2$, therefore, one should look for similarities at frequencies also spaced by a factor of two.

\section{B. Input Return Loss}

The return loss of the Sierpinski monopole was measured in an HP-8510B network analyzer in the $0.05-16-\mathrm{GHz}$ frequency range. The antenna was also simulated on a connection machine CM-5 using an FDTD algorithm. Furthermore, five bow ties of the same sizes as each one of the gaskets on the Sierpinski antenna $(89,44.5,22.3,11.1$, and $5.5 \mathrm{~mm})$ were measured to compare the results. Fig. 2 shows the input
TABLE I

Main Parameters of the Measured Sierpinski Monopole

\begin{tabular}{|c|c|c|c|c|c|}
\hline $\bar{n}\left(\right.$ band $\left.n^{o}\right)$ & $f_{n}(G H z)$ & Bwidth (\%) & $L_{r} \quad(d B)$ & 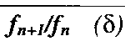 & $\overline{h_{n} / \lambda_{n}}$ \\
\hline 1 & 0.52 & 7.15 & 10 & 3.50 & 0.153 \\
\hline 2 & 1.74 & 9.04 & 14 & 2.02 & 0.258 \\
\hline 3 & 3.51 & 20.5 & 24 & 1.98 & 0.261 \\
\hline 4 & 6.95 & 22 & 19 & 2.00 & 0.257 \\
\hline 5 & 13.89 & 25 & 20 & - & 0.255 \\
\hline
\end{tabular}

reflection coefficient relative to $50 \Omega$ of the five-monopole bow ties together with the Sierpinski monopole (the plot corresponding to the Sierpinski antenna appears at the bottom of the figure); Table I summarizes the main parameters derived from that plot.

The five bands on Table I correspond to the five voltage standing wave ratio (VSWR) minimums of the antenna. The frequencies corresponding to such minimums appear in the second column $\left(f_{n}\right)$. The third column describes the relative bandwidth at each band for VSWR $<2$, the fourth one the input return loss $\left(L_{r}\right)$, the fifth one represents the frequency ratio between two adjacent bands, and the sixth one the ratio between the height of each of the five subgasket and the corresponding band frequency. As it was described in [20], the Sierpinski monopole presents a log-periodic behavior with five bands approximately spaced by a factor $\delta=2$; the antenna keeps a notable degree of similarity through the bands, with a moderate bandwidth $(\sim 21 \%)$ at each one. To get a better insight on the log-periodic behavior of the antenna, the input impedance is also shown in a semilogarithmic scale (Fig. 3).

It can be seen that the antenna is matched approximately at frequencies

$$
f_{n} \approx 0.26 \frac{c}{h} \delta^{n}
$$

where $c$ is the speed of light in vacuum, $h$ is the height of the largest gasket, $\delta$ the $\log$ period $(\delta \approx 2)$, and $n$ a natural number. The bands are slightly deviated from those in [20], where $h / \lambda \approx 0.29$. This is related to the thicker substrate ( $h=1.588 \mathrm{~mm}$ as opposed to $h=0.127 \mathrm{~mm}$ ) and its higher permitivity $\left(\varepsilon_{r}=2.5\right.$ as opposed to $\left.\varepsilon_{r}=2.17\right)$, which makes the whole structure appear slightly electrically longer [27] than the one with the thinner, lower permitivity substrate. Anyway, such a behavior is clearly different to that of the bow-tie monopole, which has the first minimum VSWR at $h / \lambda=0.17$ and the corresponding higher order modes periodically spaced by a frequency gap of $\Delta f=0.44 c / h \mathrm{~Hz}$; that is

$$
f_{n} \approx(0.17+0.44 n) \frac{c}{h} \text {. }
$$

This is a comparable result to the classical one from Brown and Woodward [28] who measured (from a thinner bow tie) a first match at $0.194 c / h$ and a second one at $0.43 c / h$ from the first.

The second match of the bow-tie antenna is always better than the first one ( $L_{r}>15 \mathrm{~dB}$ as opposed to $L_{r}=8 \mathrm{~dB}$ ), which might suggest that it can have a more significant effect on the Sierpinski behavior. Thus, if we assimilate each of 

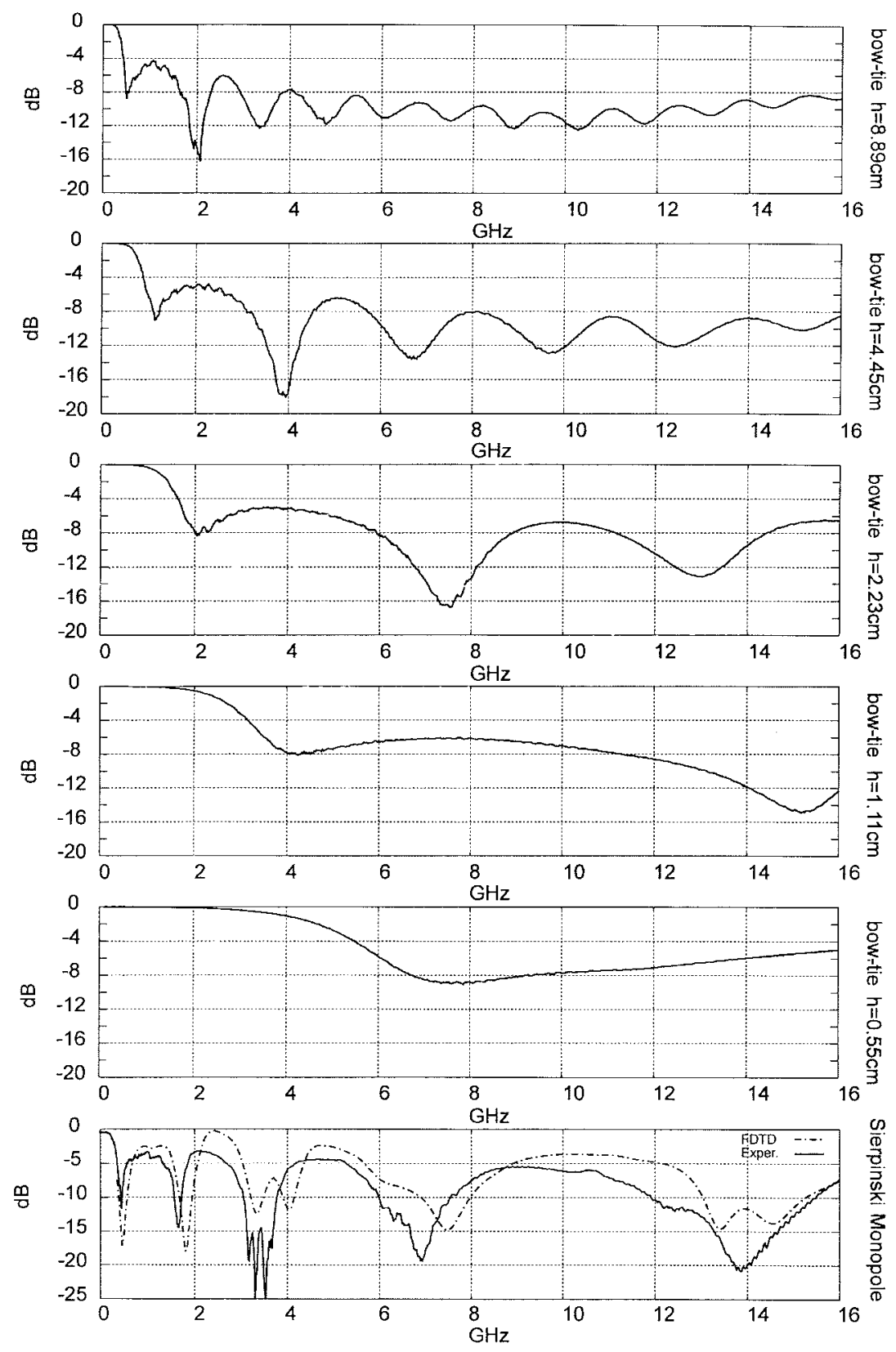

Fig. 2. Input reflection coefficient (referred to $50 \Omega$ ) of five bow ties at scaled as each one of the five subgaskets on the Sierpinski monopole (89, 44.5 , $22.3,11.1$, and $5.5 \mathrm{~mm}$ ). The bottom plot corresponds to the input reflection coefficient of the Sierpinski monopole.

the lowest subgaskets (circled in dashed lines in Fig. 1) to a bow tie, the Sierpinski bands could correspond to the second one of the triangular antennas. The frequency shift toward the origin experimented by the fractal antenna, with respect to the triangular, can be related to the capacitive loading of the upper subgaskets. It is also interesting to notice that the similarity and periodicity are lost in the lower bands where the input return loss and $h / \lambda$ ratio are closer to those of the bow tie. This fact can be related to the antenna truncation since the structure is not an ideal fractal constructed after an infinite number of iterations. Although an ideal fractal shape is self similar [8] at an infinite number of scales, a feasible implementation of the structure only keeps a certain degree of similarity over a finite number of scales, which limits the number of operating bands. Therefore, in the low-frequency region, the lowest matched frequency is shifted closer to that of the first one of a bow tie the same size as the whole Sierpinski antenna. Such a truncation effect becomes more apparent when one looks at the current distribution over the antenna (Section IV).

\section{Radiation Patterns}

The main cuts $\left(\varphi=0^{\circ}, \varphi=90^{\circ}, \theta=0^{\circ}\right)$ of the fractal monopole radiation patterns where measured in a $10 \times 7.5$ $\times 7.5 \mathrm{~m}$ anechoic chamber. The cuts where measured at the four upper bands (Fig. 4), where similar patterns among bands should be expected. As it was shown ${ }^{1}$ in [20], the patterns do keep a certain degree of similarity: the $\varphi=0^{\circ}$ patterns for the

\footnotetext{
${ }^{1}$ There was a misprint in the results published in [20]: the $\varphi=0$ and $\varphi=90$ cuts should be swapped.
} 

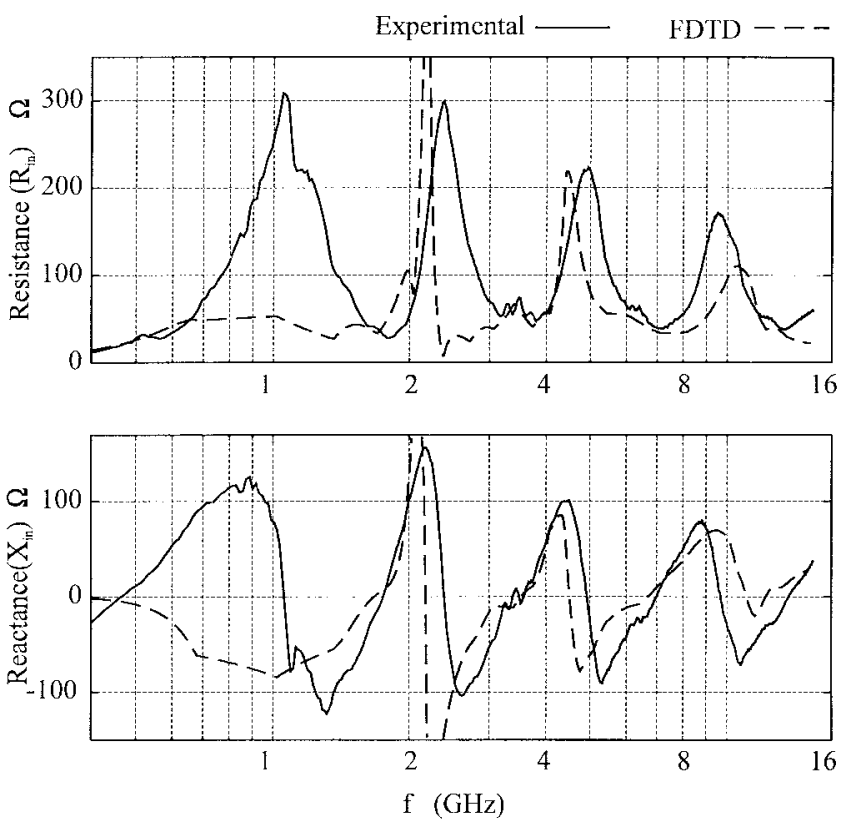

Fig. 3. Input resistance (top) and reactance (bottom) of the five-iteration Sierpinski monopole. Frequency is shown in logarithmic scale to stress the log-periodic behavior of the antenna.

$E_{\theta}$ component are characterized by a two-lobe structure with a dip located approximately at an elevation angle of $30^{\circ}$; the $\varphi=90^{\circ}$ patterns display a monopole-like pattern with a dip approximately at the same elevation angle, and the azimuth cut has an elliptic shape with a stronger radiation component toward the $x$ axis. The patterns for the $E_{\theta}$ component looked similar as well [20], although this component is less important since it is usually more than $20 \mathrm{~dB}$ below the other one.

These results are clearly different from those of a typical single-band antenna such as a monopole or a bow-tie antenna since the Sierpinski antenna has an electrical length slightly longer than four wavelengths at the upper band and a monopole or a bow tie would have several grating lobes at such a high frequency. However, it should be noticed that the effect of the finite size of the ground plane must be taken into account when analyzing the patterns on Fig. 4 [19]. For instance, those at the upper bands show a characteristic ripple, which is due to diffraction at the edges of the plane. The variations on the ripple are faster when frequency is increased since the squared plane is obviously not self-scalable and the edges are spaced a longer distance in terms of the corresponding wavelength. Also, the expected null in the $z$-axis direction is hidden by the contribution of the antisymmetrical mode of the ground plane to the overall radiated power [19].

\section{THE SIERPINSKI DIPOLE}

\section{A. Antenna Description}

In order to properly distinguish the real influence of the Sierpinski structure on the antenna radiation patterns, a Sierpinski dipole was constructed and measured. The antenna was fed by means of a coaxial tapered balun similar to that described in [29] to balance and match the dipole through the whole $1: 16$ -
$\mathrm{GHz}$ frequency range. Basically, the balun was built by cutting open the outer wall of the coax so that a cross-sectional view showed a sector of the outer conductor removed and the round structure smoothly yielded a two-wire transmission line.

Both arms of the antenna were printed on the same substrate used in the monopole. The measurements were carried out in a roll over azimuth configuration with the balun mounted along the $y$ and $x$ axis alternatively to minimize the effect of both the rotation axis and the balun on the $\varphi=0^{\circ}$ and $\varphi=90^{\circ}$ patterns, respectively. The coordinate reference system is the same of Fig. 1. An identical scheme was used to measure an equilateral bow-tie antenna the same size as the Sierpinski one.

\section{B. Radiation Patterns}

The patterns of both the Sierpinski dipole and a bow-tie antenna of the same size $(89 \mathrm{~mm}$ was the height of each arm) can be compared on Figs. 5 and 6. Each row displays three patterns within each one of the four upper bands of the Sierpinski antenna. The cuts corresponding to the Sierpinski antenna are represented on the left-hand side of the figure, while the same cuts corresponding to the bow tie antenna are plotted on the right. Each one of the two figures displays a cut through the main planes $\left(\varphi=0^{\circ}, \varphi=90^{\circ}\right)$ for both antennas. The $\theta=90^{\circ}$ cut is not significant in this case due to the roll over azimuth measurement scheme.

It is interesting to notice the strong similarity between patterns through the four bands (rows) on the Sierpinski antenna. These similarities are specially remarkable at 2, 4, 8, and $16 \mathrm{GHz}$. Again, these results are clearly different to those of the bow-tie antenna. Although the bow-tie antenna has many matched frequencies as described in (2), each resonant mode has a different current density distribution, which is translated into a different pattern for each frequency. The behavior is, thus, more similar to that of a single-band dipole in which increasing the operating frequency results in an increment on the number of grating lobes of the pattern. Hence, we cannot either talk about a dipole or a bow-tie antenna as a multiband antenna because the patterns are not held similar through the matched frequencies. On the contrary, we must think of the Sierpinski antenna as a multiband one since both the input return loss and the radiation patterns are held similar through the bands. Nevertheless, the antenna is not frequency independent since there is a remarkable variation of both the patterns and the return loss through each log period.

When comparing the dipole patterns to the monopole ones, the nonidealities of both the balun and the ground plane can be detected. The balun does not perfectly balance the current between both arms of the dipole, consequently, the cuts over the planes orthogonal to the $X Y$ plane display a slight asymmetry. Such an asymmetry enhances radiation toward the $-z$ axis (see $\varphi=0^{\circ}$ cut at 3.6 and $7.2 \mathrm{GHz}$ ) and tends to hide some nulls of the pattern. Nevertheless, the lobe structure becomes apparent and the similarity among bands appears clearly. Furthermore, the expected nulls along the $z$ axis can be distinguished now, which supports the idea that 

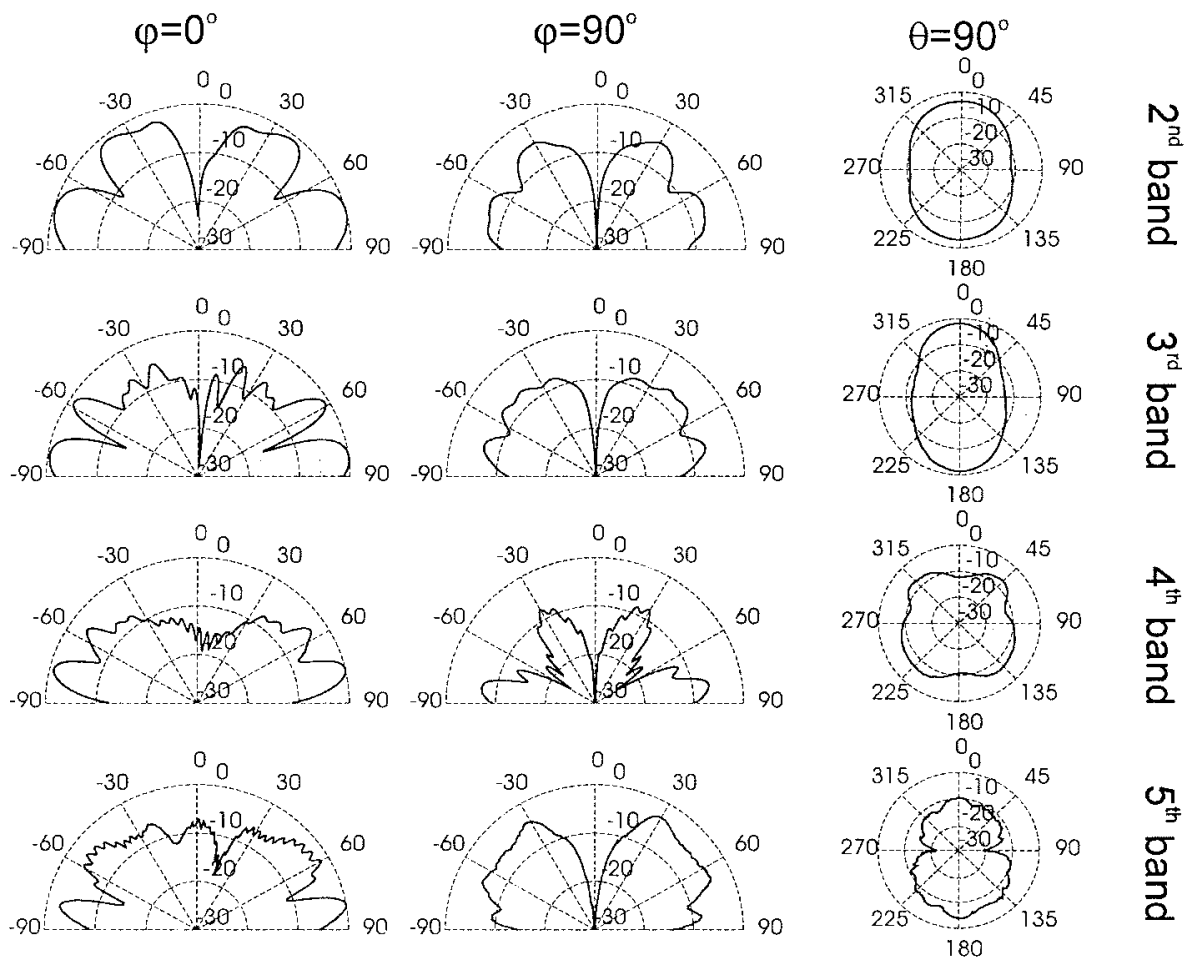

Fig. 4. Main cuts of the Sierpinski monopole radiation pattern ( $E_{\theta}$ component). From left to right, each column corresponds to one cut $\left(\varphi=0^{\circ}, \varphi=90^{\circ}, \theta=90^{\circ}\right.$ ) at each one of the four upper bands (from top to bottom $f=1.74 \mathrm{GHz}, f=3.51 \mathrm{GHz}, f=6.95 \mathrm{GHz}$, and $f=13.89 \mathrm{GHz}$ ). Each pattern is normalized with respect to its own maximum.

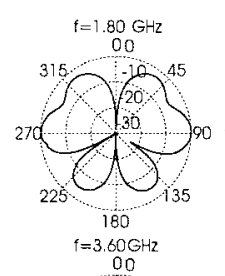

$\frac{00}{-10}+45$

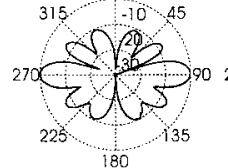

$225 \quad 180$
$\mathrm{f}=7.20 \mathrm{GHz}$

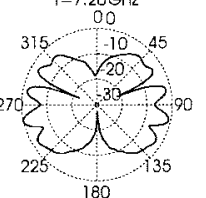

180
$f=14.40 \mathrm{GHz}$

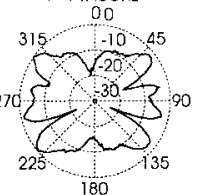

180

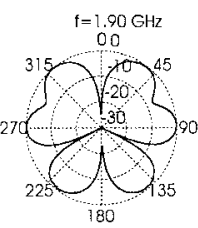

$\mathrm{f}=3.80 \mathrm{GHz}$

$\mathrm{f}=3.80 \mathrm{GHz}$
00

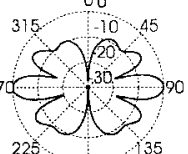

$225{ }_{180}$

$\mathrm{f}=7.60 \mathrm{GHz}$

$\mathrm{f}=7.60 \mathrm{GHZ}$
00
0
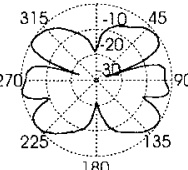

$f=15.20 \mathrm{GHz}$

$f=15.20 \mathrm{GHz}$
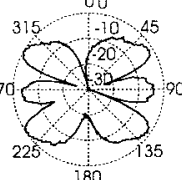

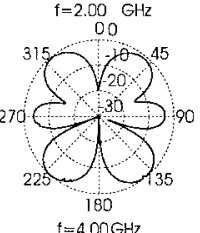

$=4.00 \mathrm{GH}$
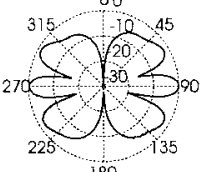

$\mathrm{t}=8.00 \mathrm{GH}$

$\mathrm{f}=8.00 \mathrm{GHz}$
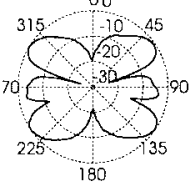

180
$\mathrm{f}=10,00 \mathrm{GHz}$
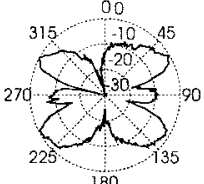

180

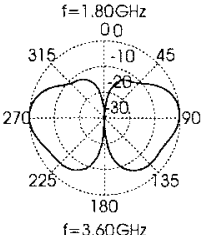

3.60G

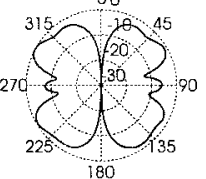

$\mathrm{f}=7.20 \mathrm{GHz}$

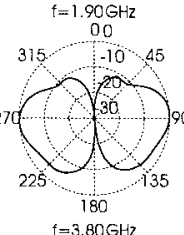

$1=3.80 \mathrm{GH}$
00

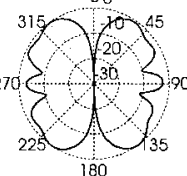

$f=7.60 \mathrm{GH}$

$.60 \mathrm{GH} 2$
$0 \mathrm{O}$
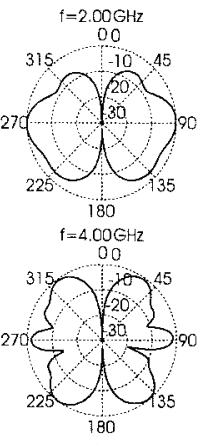

$\mathrm{f}=8.00 \mathrm{GHz}$

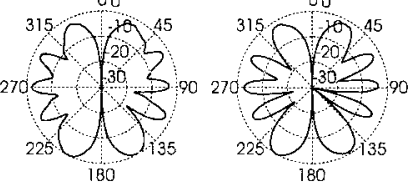

180
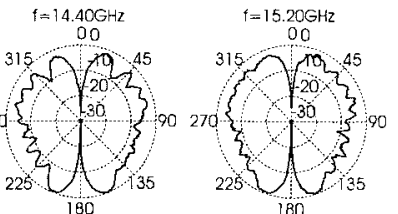

180

Fig. 5. Front to back cut $\left(\varphi=0^{\circ}\right)$ of both the (a) Sierpinski dipole radiation pattern and the (b) bow-tie antenna. The patterns correspond to the $E_{\theta}$ component. Each row displays three cuts within one band. Notice the similarity between rows (multiband behavior) as opposed to the differences between columns (not a frequency-independent behavior) on the Sierpinski antenna.

the former ripple and lobes appeared in the monopole were due to the ground plane.

Another interesting feature of the Sierpinski patterns is the characteristic three-lobe structure on the $\varphi=0^{\circ}$ cut. Such a pattern is comparable to that on the third matched frequency of the bow-tie antenna $(f \approx 3.6 \mathrm{GHz}$ for $h=89 \mathrm{~mm})$; if we compare such frequencies of the five bow ties [(1), Fig. 2] to those of the Sierpinski one (2), we can see that the third matched one on each bow tie is very close to a Sierpinski band. Hence, it seems that the fractal antenna could be operating at 

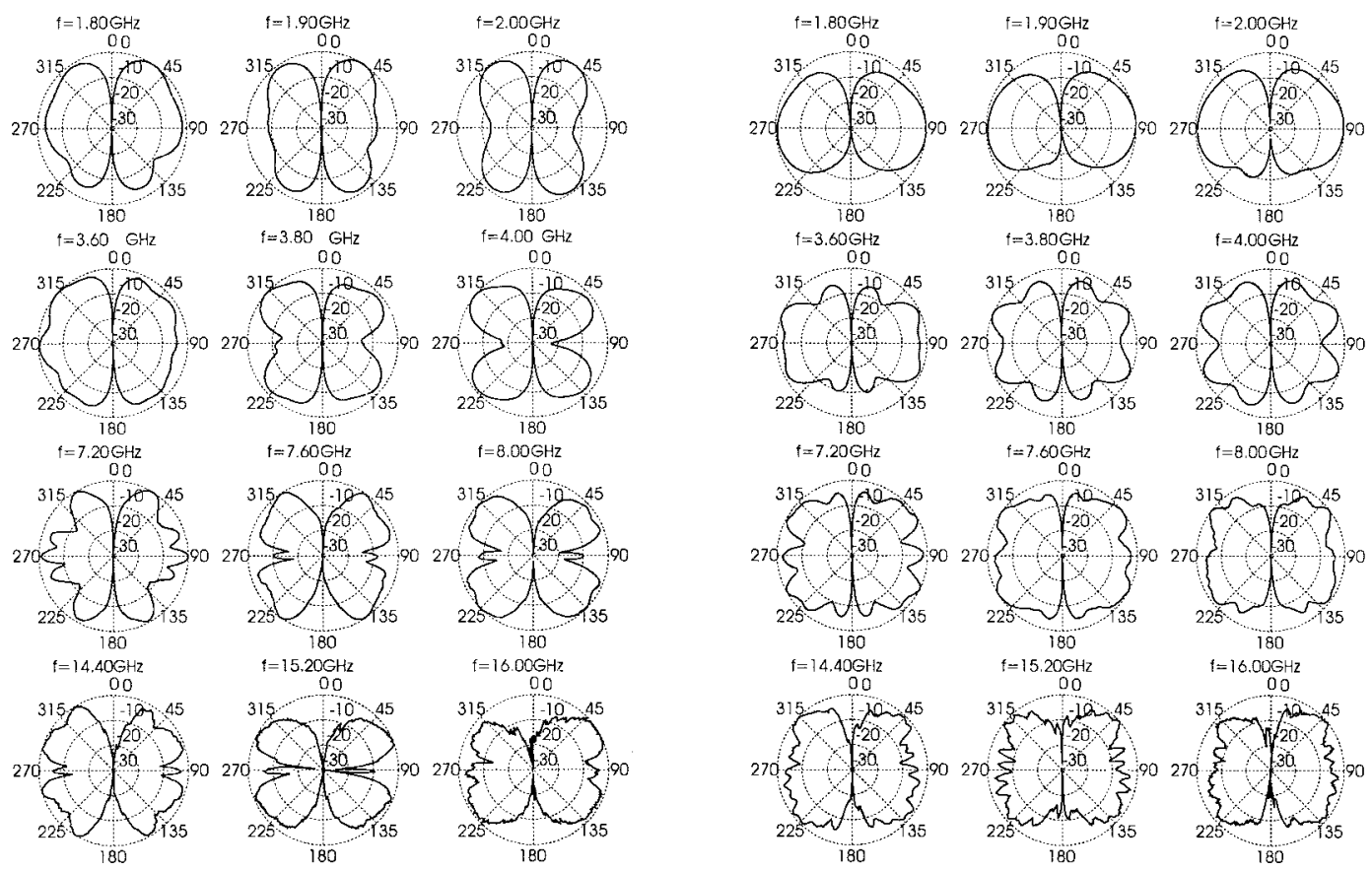

Fig. 6. Left to right cut $\left(\varphi=90^{\circ}\right)$ of both the (a) Sierpinski dipole radiation pattern and the (b) bow-tie antenna. The patterns correspond to the $E_{\theta}$ component. Each row displays three cuts within one band. Notice the similarity between rows (multiband behavior) as opposed to the differences between columns (not a frequency-independent behavior) on the Sierpinski antenna.

each band in a combination of two modes corresponding to two bow ties at different scales, the larger working on its third resonance, and the smaller on its second one.

\section{CURRENT DENSITY OVER THE SierPinski ANTENNA}

In order to get a deeper physical insight on the behavior of the Sierpinski antenna, an FDTD algorithm was developed to calculate the current density distribution over the fractal surface. The algorithm was run on a connection machine CM5 using a $32 \times 256 \times 256$ cell space. The Yee cell dimensions were $\Delta x=0.463 \mathrm{~mm}, \Delta y=0.267 \mathrm{~mm}, \Delta z=0.463 \mathrm{~mm}$, and second-order Mur absorbing boundary conditions [30] were used.

A sinusoidal signal at each of the four upper bands was used to excite the antenna. After the transient period, the current density maximum at each point was detected. On the left column of Fig. 7, the magnitude of the density current distribution at each band over the whole antenna surface is given (linear scale, arbitrary units). The right column presents an expanded view of the region where most of the current is concentrated at each frequency. All of them are scaled by a factor of two, the same scale factor existing among bands. It is interesting to notice that the current density distributions on the right column are very similar among them; that is especially true if the effect of the smaller wholes at the lower bands are neglected. This is a reasonable approximation since such wholes are small compared to the wavelength and the current density is lower at the regions where the wholes are located. Therefore, the similarity among the patterns shown in the previous section can now be explained: at each band the current concentrates over a properly scaled substructure on the antenna which has the main contribution to the overall radiation pattern. It should be noticed that this region is smaller when frequency is increased and that the current does not reach the top of the antenna at the highest bands and a large area of the structure becomes this way effectively disconnected. This phenomena is equivalent to the active region found in the log periodic arrays described in the early sixties by Carrel and Mayes [14], [18]. Now, an additional explanation on the behavior of the fractal antenna can be given: when an electromagnetic wave is fed through the tip of the antenna it starts to propagate over the structure toward the flat end of the gasket. When the wave finds a cluster comparable in size to its wavelength it becomes radiated; this way the power of the traveling wave is lost and no current reaches the end of the antenna. The structure has many discontinuities that enhance radiation and emphasizes such a process. If the wavelength is long, the small triangles of the shape contribute less to the overall radiation and the current can travel further over it; then a larger active region can be reached (which will have a similar shape to that of a shorter wavelength) and a similar radiation pattern for such a lower band will be obtained.

Finally, the plots on Fig. 7 can explain the truncation effect described in Section II. Although it is difficult to precisely put bounds to the active region, it actually covers a wider area than the encircled regions of Fig. 1; at least an additional scale level should be taken into account to properly model the antenna behavior. Thus, at the lowest band the whole antenna is not large enough, the active region is distorted and the antenna looses the self-similarity properties with respect the higher bands. Also, it is interesting to notice that the active region covers an area longer ( $z$ direction) than the operating wavelength which can explain the similarity of the $\varphi=0^{\circ}$ pattern to that of a $1.5-\lambda$ dipole [19]. 


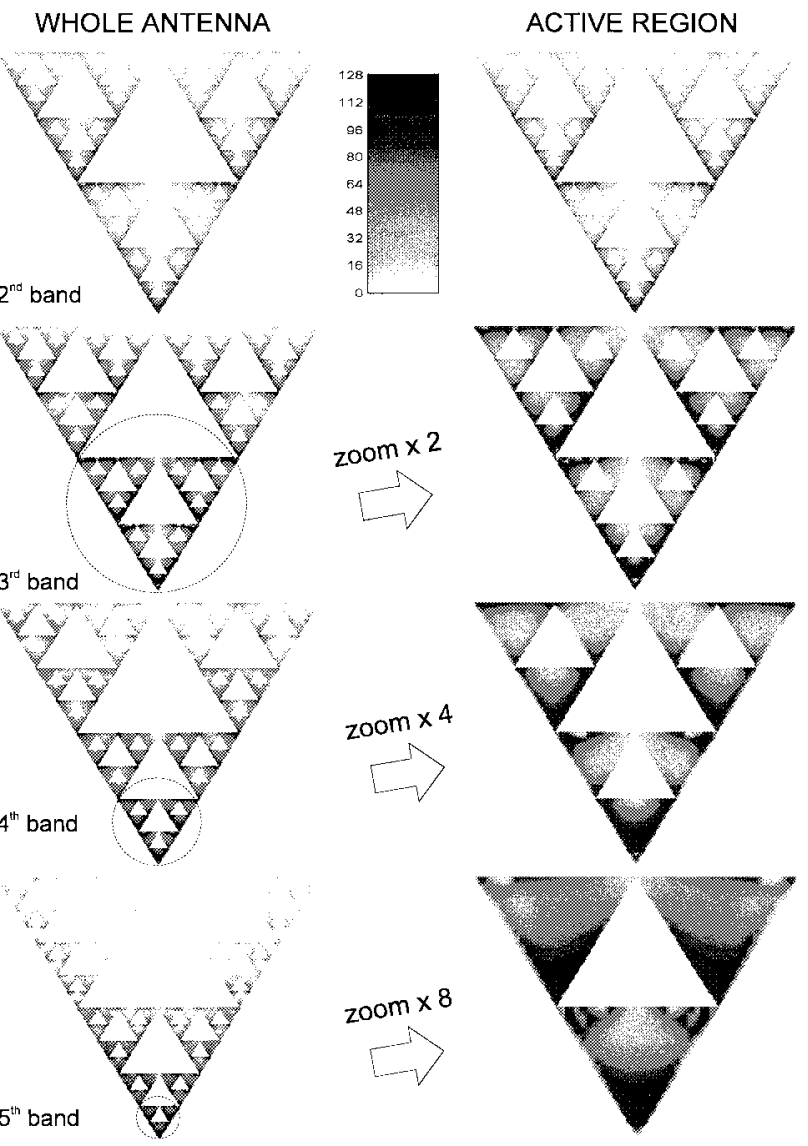

Fig. 7. Current density (magnitude) over the Sierpinski dipole computed by means of the FDTD algorithm. The left column displays the whole antenna at the four upper bands while the right column shows a zoomed view of the active region at each band. Notice the similarity between the distributions over the active region at each band.

\section{CONCLUSIONS}

Both experimental and numerical results on the Sierpinski antenna have been presented. All of them describe a multiband behavior of the fractal antenna. This behavior is consistent from the input return loss and the radiation patterns points of view. The bands are log-periodically spaced by a factor of two, the same scale factor existing among similar structures on the fractal shape. Thus, it can be concluded that the self-similarity properties of the fractal structure are translated into its electromagnetic behavior. The current density over the antenna has been also calculated by means of an FDTD algorithm. The input return loss calculated using this algorithm is consistent with the experimental one. The density current distributions have a similar shape among bands as well, thus explaining the similarity among the patterns found experimentally. Such distributions describe an active region over the antenna that self scales with frequency and allows a similar behavior of the antenna through the bands.

\section{ACKNOWLEDGMENT}

The authors would like to thank Dr. P. E. Mayes of the University of Illinois at Urbana-Champaign and Dr. D. L. Jaggard of the University of Pennsylvania, Philadelphia, for encouraging this work. They would also like to thank
R. Bartolomé, A. Hijazo, F. Benítez, and X. Garcia for contributions to the measurements and numerical results. The numerical results were obtained at the Centre National de Calcul Parallele en Sciences de la Terre, Paris, France.

\section{REFERENCES}

[1] D. L. Jaggard, "On fractal electrodynamics," in Recent Advances in Electromagnetic Theory, H. N. Kritikos and D. L. Jaggard, Eds. New York: Springer-Verlag, 1990, pp. 183-224.

[2] Y. Kim and D. L. Jaggard, "The fractal random array," Proc. IEEE, vol. 74, pp. 1278-1280, Sept. 1986.

[3] D. L. Jaggard and T. Spielman, "Triadic cantor target diffraction," Microwave Opt. Technol. Lett., vol. 5, pp. 460-466, Aug. 1992.

[4] D. L. Jaggard, "Prologue to special section on fractals in electrical engineering," Proc. IEEE, vol. 81, pp. 1423-1427, Oct. 1993.

[5] M. M. Beal and N. George, "Features in the optical transforms of serrated apertures and disks," J. Opt. Soc. Amer., vol. 6, no. 12, pp. 1815-1826, Dec. 1989.

[6] Y. Kim, H. Grebel, and D. L. Jaggard, "Diffraction by fractally serrated apertures," J. Opt. Soc. Amer., vol. 8, no. 1, pp. 20-26, Jan. 1991.

[7] X. Sun and D. L. Jaggard, "Wave interactions with generalized cantor bar fractal multilayers," J. Appl. Phys., vol. 70, no. 5, pp. 2500-25007, Sept. 1991.

[8] H. O. Peitgen, H. Jürgens, and D. Saupe, Chaos and Fractals. New York: Springer-Verlag, 1990.

[9] M. F. Barnsley, R. L. Devaney, B. B. Mandelbrot, H. O. Peitgen, D. Saupe, R. F. Voss, Y. Fisher, and M. Mc Guire, The Science of Fractal Images. New York: Springer-Verlag, 1988

[10] H. Jones, D. E. Reeve, and D. Saupe, Fractals and Chaos, A. J. Crilly, R. A. Earnshaw, and H. Jones, Eds. New York: Springer-Verlag, 1990.

[11] B. B. Mandelbrot, The Fractal Geometry of Nature. San Francisco, CA: Freeman, 1983

[12] C. Goutelard, "Fractal theory of large arrays of lacunar antennas," in Electromagn. Wave Propagat. Panel Symp. (AGARD-CP-528), France, June 1992, pp. 35/1-35/15.

[13] C. Puente and R. Pous, "Fractal design of multiband and low side-lobe arrays," IEEE Trans. Antennas Propagat., vol. 44, pp. 1-10, May 1996

[14] P. E. Mayes, "Frequency-independent antennas and broad-band derivatives thereof," Proc. IEEE, vol. 80, pp. 103-112, Jan. 1992.

[15] P. E. Mayes, G. A. Deschamps, and W. T. Patton, "Backward-wave radiation from periodic structures and application to the design of frequency-independent antennas," Proc. IRE, vol. 49, pp. 962-963, May 1961.

[16] G. A. Deschamps and J. D. Dyson, "The logarithmic spiral in a singleaperture multimode antenna system," IEEE Trans. Antennas Propagat., vol. AP-19, pp. 90-96, Jan. 1971.

[17] V. H. Rumsey, Frequency Independent Antennas. New York: Academic, 1966

[18] R. L. Carrel, "Analysis and design of the log-periodic dipole antenna," Doctoral dissertation, Dept. Elect. Eng., Univ. Illinois, UrbanaChampaign, 1961.

[19] H. Jasik, Antenna Engineering Handbook. New York: McGraw-Hill, 1961, pp. 2.10-2.13.

[20] C. Puente, J. Romeu, R. Pous, X. Garcia, and F. Benitez, "Fractal multiband antenna based on the Sierpinski gasket," Electron. Lett., vol. 32, pp. 1-2, Jan. 1996.

[21] C. Puente, J. Romeu, R. Bartolomé, and R. Pous, "Perturbation of the Sierpinski antenna to allocate operating bands," Electron. Lett., vol. 32, pp. 2186-2188, Nov. 1996.

[22] C. Puente, J. Claret, F. Sagués, J. Romeu, M. Q. López-Salvans, and R. Pous, "Multiband properties of a fractal tree antenna generated by electrochemical deposition," Electron. Lett., vol. 32, pp. 2298-2299, Dec. 1996.

[23] C. Puente, "Fractal antennas," Ph.D. dissertation, Dept. Signal Theory Communicat., Universitat Politécnica de Catalunya, June 1997.

[24] N. Cohen, "Fractal antennas: Pt. 2," Communicat. Quart., pp. 53-66, Summer 1996.

[25] D. H. Werner and P. L. Werner, "Frequency independent features of self-similar fractal antennas," Radio Sci., vol. 31, no. 6, pp. 1331-1343, Nov./Dec. 1996.

[26] W. Sierpinski, "Sur une courbe dont tout point est un point de ramification," C. R. Acad., Paris 160 302, 1915.

[27] A. Rubio, A. Salinas, R. Gómez, and I. Sánchez, "Time-domain analysis of dielectric-coated wire antennas and scatterers," IEEE Trans. Antennas Propagat., vol. 42, pp. 815-819, June 1994. 
[28] G. H. Brown and O. M. Woodward, "Experimentally determined radiation characteristics of conical and triangular antennas," RCA Rev., pp. 425-452, Dec. 1952.

[29] J. W. Duncan and V. P. Minerva, "100: 1 bandwidth balun transformer," in Proc. IRE, vol. 48, pp. 156-164, Feb. 1960.

[30] G. Mur, "Absorbing boundary conditions for the finite-difference approximation of the time-domain electromagnetic-field equations," IEEE Trans. Electromagn. Compat., vol. EMC-23, pp. 377-382, Nov. 1981.

Carles Puente-Baliarda (S'91-M'93) was born in Badalona, Spain, In 1968. He received the Ingeniero degree in telecommunications engineering from the Polytechnic University of Catalonia (UPC), Barcelona, Spain, in 1992, the M.S. degree from the University of Illinois at Urbana-Champaign (UIUC), in 1994, and the Ph.D. degree from the UPC, in 1997.

In 1992, he received a grant from the Interdepartmental Commission for Research and Technological Innovation (CIRIT) of the Catalan Government and joined the Electro Optic Systems Laboratory (EOSL) at the UIUC, where he worked in laser velocimetry and lidar techniques until December 1993. Since 1994, he has been an Assistant Professor at the Department of Signal Theory and Communications at the Polytechnic University of Catalonia. His current research interests are fractal antennas, lidar, laser systems, and optical communications.

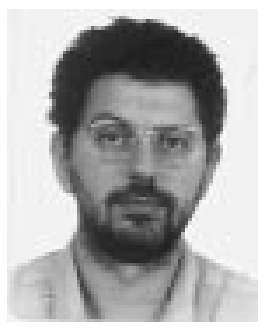

Jordi Romeu (S'89-M'91) was born in Barcelona, Spain, in 1962. He received the Ingeniero and Doctor Ingeniero degrees in telecommunication engineering, both from the Polytechnic University of Catalonia (UPC), Barcelona, Spain, in 1986 and 1991, respectively.

In 1985, he joined the Electromagnetic and Photonic Engineering Group of the Signal Theory and Communications Department at the UPC. Currently, he is an Associate Professor at the same university, where he is engaged in research in antenna near-field measurements, antenna diagnostics, and antenna design.

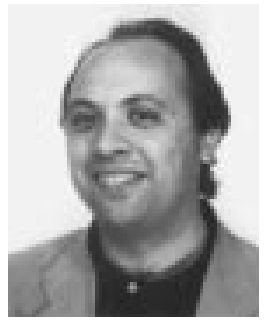

Rafael Pous (S'89-M'93) was born in Barcelona, Spain, in 1964. He received the Ingeniero degree in telecommunications engineering and the Licenciado degree in computer science from the Polytechnic University of Catalonia (Barcelona), in 1988, the M.S. degree in electrical engineering from the University of Massachusetts, Amherst, in 1989, and the Ph.D. degree in electrical engineering from the University of California, Berkeley, in 1992.

In 1993, he joined the faculty of the Department of Telecommunications Engineering at the Polytechnic University of Catalonia, Barcelona, Spain, where he is currently employed. His research interests are in the area of computational electromagnetics and applied superconductivity.

Dr. Pous was awarded the Fulbright Scholarship and the Schlumberger Fellowship during his studies.

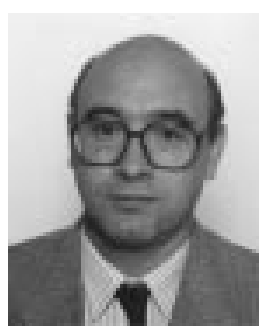

Angel Cardama (S'67-M'73) was born in Santiago de Compostela, Spain, on May 13, 1944. He received the Ingeniero de Telecommunicación degree from the Universidad Politécnica de Madrid, in 1968, and the Sc.M. and Ph.D. degrees in electrical engineering from Brown University, Providence, RI, in 1970 and 1973, respectively.

In 1972, he joined the faculty of the Telecommunication Engineering School at the Polytechnic University of Catalonia, Barcelona, Spain, where he holds the position of Professor. His research interests range from the development of analytical and numerical techniques in electromagnetics to the design of microwave imaging systems and radar and communication antennas. 